\title{
1 Surface electromyographic analysis of the serratus anterior during bench press variations
}

\author{
Kyle L. Rogers, ${ }^{1}$ Alex Caravan, ${ }^{1}$ John O. Scheffey, ${ }^{1}$ Kyle J. Boddy ${ }^{1}$
}

${ }^{1}$ Driveline Baseball, Kent, WA, USA

\begin{abstract}
Purpose: Bench press variations that elicit greater serratus anterior muscle activation may be preferable for improving scapula stability and avoiding injury in overhead throwing athletes. Here we compare serratus anterior activity during dumbbell and barbell bench press using surface electromyography (EMG). four sets of bench press-light (43 kg) and heavy (61 kg) dumbbell and barbell-with five repetitions each. Each pitcher was instructed in proper technique and rested for 90 seconds contraction (for normalization) and during each set.

Results: Dumbbell sets showed significantly greater serratus anterior activation than barbell sets at both weights (weight-normalized mean EMG amplitude, 44.7 versus $38.2 ; p=.005$ ). anterior muscle strength.

Keywords

overhead throwing athlete, dumbbell, barbell, scapular stability, activation

DOI

http://dx.doi.org/10.31236/osf.io/cgfwh
\end{abstract}

Methods: Forty college and professional baseball pitchers (men, age $22.6 \pm 2.5$ years) performed between sets. Surface electrodes were used to record serratus anterior activity during isometric Conclusion: This suggests that dumbbell press is preferable to barbell press for building serratus

\section{Citation}

Rogers KL, Caravan A, Scheffey JO, Boddy KJ (2019). Surface electromyographic analysis of the serratus anterior during bench press variations. SportRxiv. doi: .31236/osf.io/cgfwh 


\section{Introduction}

Scapula stability and shoulder muscle strength are primary concerns for overhead throwing athletes such as baseball pitchers. Instability of the scapula and weakness of overhead stabilizing muscles can cause poor force generation during throwing and vulnerability to shoulder injury. ${ }^{1}$ The serratus anterior is critical to upward scapula rotation and scapula stabilization during overhead movements, and the strength of this muscle can be the limiting factor in overall scapula movement. ${ }^{2}$ Bench press exercises target the serratus anterior and are commonly used by pitchers to improve upper-body strength and stability. Variations of the bench press that elicit greater serratus anterior activation may be preferable for improving scapula stability, minimizing movement dysfunction, and avoiding injury. ${ }^{3}$

Surface electromyography (EMG) has been used to study the activation of large muscles in the upper extremity during bench press and related exercises..$^{4-6}$ Surface EMG measures the electric potential across skeletal muscles in response to muscle stimulation. The medial deltoid shows greater activation during barbell bench press than during bench press using a Smith machine. 4 The serratus anterior shows greater activation during the "push-up plus" and "dynamic hug" than during other low-resistance rehabilitation exercises, ${ }^{5}$ and greater activation during barbell press than during standard push-ups or wall push-ups. ${ }^{6}$ However, there has been no comparison of the effects of different bench press variations on serratus anterior activity.

In this study we measured serratus anterior activity during barbell and dumbbell bench press at two weights ( 43 and $61 \mathrm{~kg}$, or 95 and $135 \mathrm{lb}$ ) using surface EMG (Figures 1 and 2). We hypothesized that dumbbell press-the more unstable of the two exercises-would elicit greater serratus anterior activity than barbell press regardless of weight.

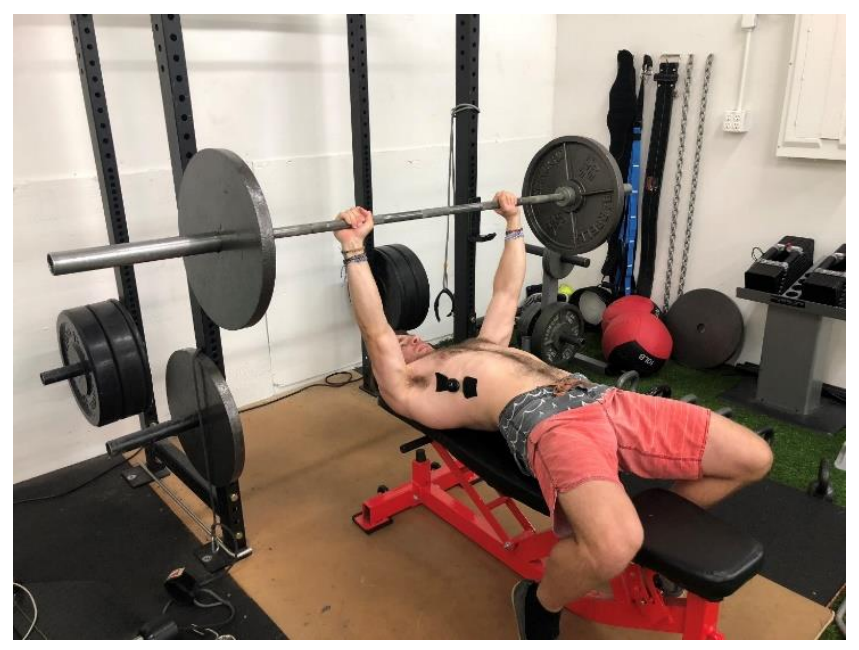

Figure 1 Caption: An example of a subject performing barbell bench press with 135 pounds 


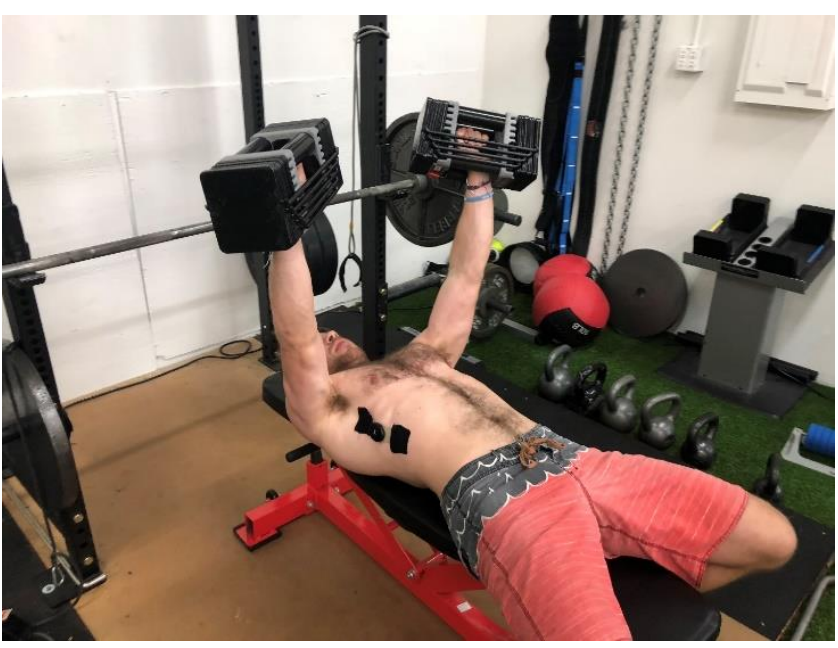

Figure 2 Caption: An example of a subject performing dumbbell bench press with 67.5 pound dumbbells

\section{Methods}

\subsection{Subjects}

Forty healthy college and professional baseball pitchers (age, $22.6 \pm 2.5$ years; height, $185.4 \pm$ $6.4 \mathrm{~cm}$; body weight, $93.6 \pm 7.8 \mathrm{~kg}$ ) were recruited for the study. The following inclusion criteria were used: men, age 18-40 years, and able to bench press $61 \mathrm{~kg}$ using barbell and dumbbell techniques without pain or discomfort in the upper extremities (including forearm, elbow, shoulder, and neck) during or after exercises. Subjects were provided a verbal explanation of the study, and were given an Informed Consent form to review and sign. If pain or discomfort was experienced at any point during the trials, the participant ceased exercises and the subject's data was discarded. The study was approved by an independent institutional review board (Hummingbird IRB; IRB\# 2018-17).

\subsection{Procedures}

The subjects were divided into four groups of 10 using a single-blind block randomization process; each group performed four sets of bench press exercises (described below) in a different order. Subjects performed no high-intensity exercise for $24 \mathrm{~h}$ before the EMG-monitored exercises. Bench press exercises were performed after a warm-up, which consisted of lowintensity foam rolling and upper-body mobility stretches.

Surface EMG data were collected as described previously.7 Raw EMG signals were collected at 1,000 Hz using a Somaxis Cricket EMG sensor and Chirp for Cricket app (Somaxis Inc., San Francisco, CA). Skin application sites were cleaned and sensors were applied vertically across a standardized site on the serratus anterior on both the left and right sides using kinesiology tape (RockTape, Inc., Campbell, CA), with 5-6 inches between sensors (Figures 3 and 4). 


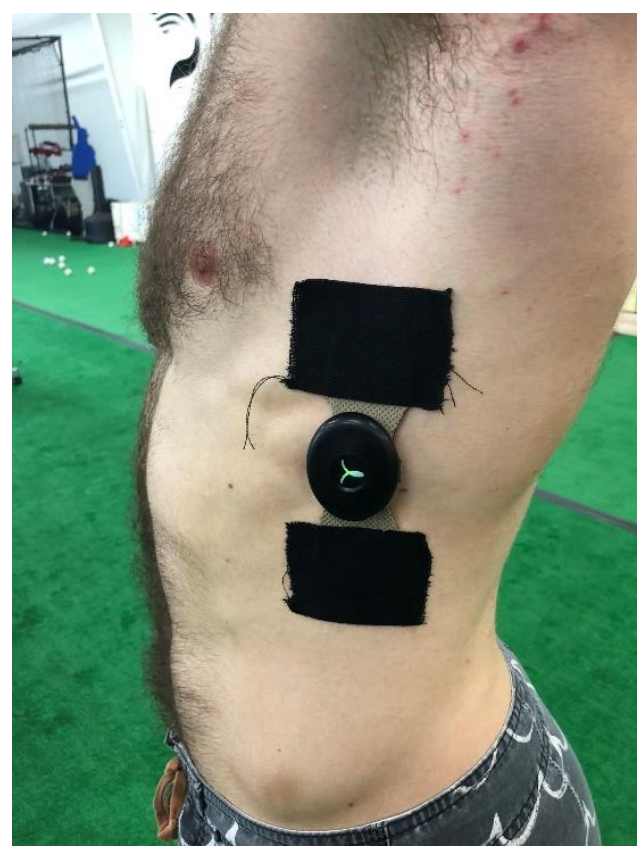

Figure 3 Caption: Application of the Somaxis EMG sensor to the right serratus anterior of the subject

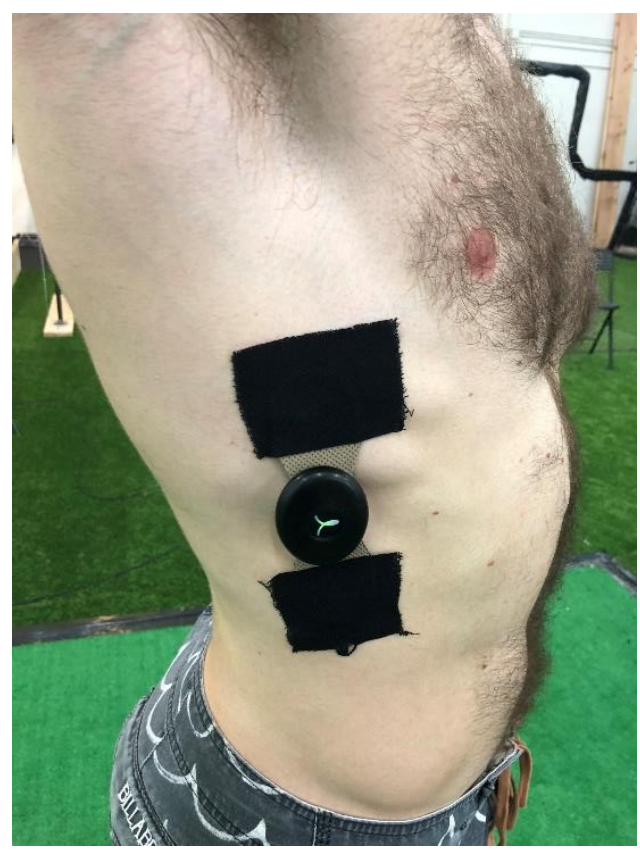

Figure 4 Caption: Application of the Somaxis EMG sensor to the left serratus anterior of the subject

To normalize EMG signals by muscle strength, the maximum voluntary isometric contraction (MVIC) of the serratus anterior on both sides of each subject was measured before bench press exercises. Subjects were instructed to raise their right arm to $125^{\circ}$ of shoulder abduction in the sagittal plane, as measured using a goniometer. (Manual resistance at this 
shoulder abduction angle causes greater serratus anterior excitation than at other angles. ${ }^{8}$ ) Subjects were then instructed to resist a downward applied to the humerus using a hand-held dynamometer (Lafayette Instrument Co., Lafayette, IN) until the goniometer read 5 lbs to obtain the MVIC EMG signal. Tests were roughly 3-4 seconds long and performed on first the right arm (Figures 5 and 6) then the left arm (Figures 7 and 8), with a ten second rest in between.

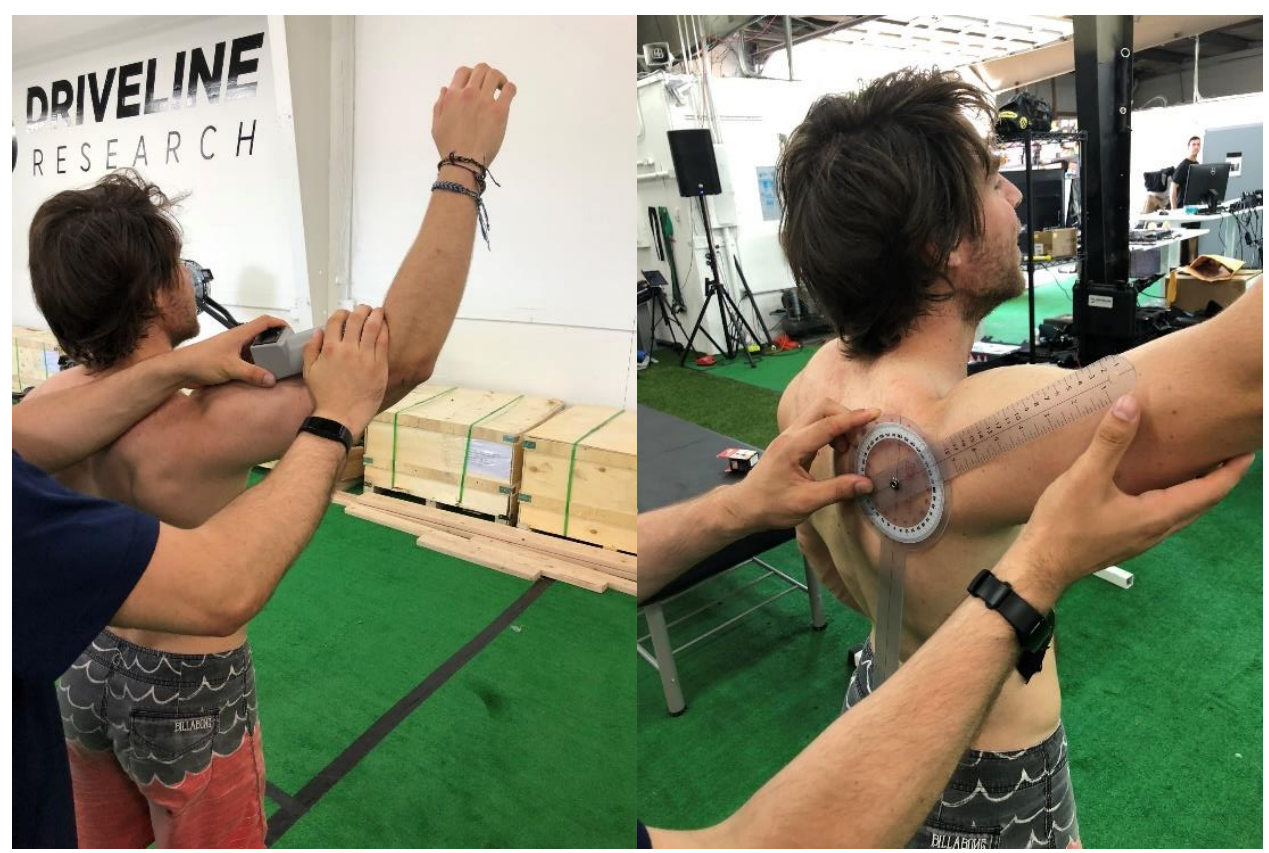

Figure 5 and 6 Captions: Measurement of the first MVIC position with the goniometer and first MVIC test done on the subject

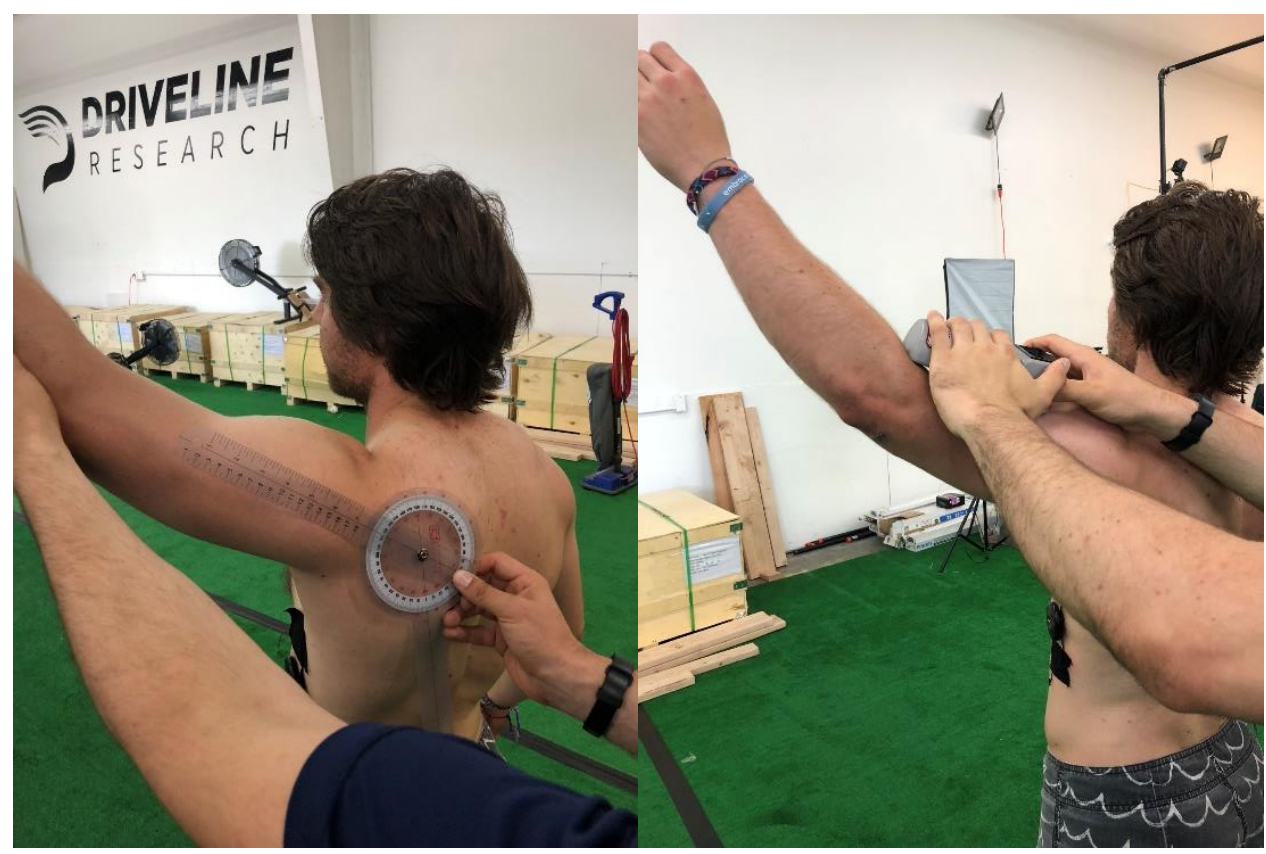

Figure 7 and 8 Captions: Measurement of the second MVIC position with the goniometer and second MVIC test done on the subject 
Subjects were instructed to engage the latissimus dorsi muscles throughout the bench press exercises, and to perform the sets at a moderate pace and intensity. Subjects performed four sets of bench press-light and heavy dumbbell and barbell-with five repetitions each and 90 seconds of rest between sets. The weights used in the four sets were the following:

1. Light dumbbells: two $21.5 \mathrm{~kg}(47.5 \mathrm{lb})$ dumbbells; total weight: $43.1 \mathrm{~kg}$ (95 lb)

2. Light barbells: two $11.3 \mathrm{~kg}$ (25 lb) plates on a $20.4 \mathrm{~kg}$ (45 lb) barbell; total weight: $43.1 \mathrm{~kg}$ (95 lb)

3. Heavy dumbbells: two $30.6 \mathrm{~kg}(67.5 \mathrm{lb})$ dumbbells; total weight: $61.2 \mathrm{~kg}(135 \mathrm{lb})$

4. Heavy barbells: two $20.4 \mathrm{~kg}(45 \mathrm{lb})$ plates on a $20.4 \mathrm{~kg}(45 \mathrm{lb})$ barbell, total weight: 61.2 $\mathrm{kg}(135 \mathrm{lb})$

These weights were chosen as moderate loads for the subjects: most subjects were able to bench press well above $61 \mathrm{~kg}$. These types of loads were common in the majority of the sample athlete's strength programs. The order of the sets was varied between the four groups as shown in Table 1.

Table 1 Sequence of sets for each group

\begin{tabular}{cllll}
\hline Group & Set 1 & Set 2 & Set 3 & Set 4 \\
\hline 1 & $\begin{array}{l}\text { Light barbell } \\
\text { Light } \\
\text { dumbbell }\end{array}$ & $\begin{array}{l}\text { Light } \\
\text { dumbbell }\end{array}$ & Heavy barbell & Heavy dumbbell \\
2 & $\begin{array}{l}\text { Light barbell } \\
\text { Light } \\
\text { dumbbell }\end{array}$ & Heavy dumbbell & Heavy barbell \\
& $\begin{array}{l}\text { Light barbell } \\
\text { Light } \\
\text { dumbbell }\end{array}$ & Light barbell & Heavy barbell & Heavy dumbbell
\end{tabular}

\subsection{Data Processing}

Surface EMG data were processed as described previously.7 A tenth-order band-pass Butterworth filter with a lower cutoff frequency of $10 \mathrm{~Hz}$ and higher cutoff frequency of $450 \mathrm{~Hz}$ was used. ${ }^{9}$ The lower cutoff was used to remove electrical noise, wire sway, and biological artifacts; the higher cutoff was used to remove tissue noise at electrode sites. ${ }^{10}$ The output was rectified, then smoothed using a moving average with a window size of 200 to further separate signal from noise. ${ }^{11}$ Serratus anterior MVIC EMG values were identified and used to normalize bench press EMG data for both sides of each subject to provide better comparisons between subjects. ${ }^{12,13}$

\subsection{Statistical Analysis}

Statistical analysis was performed on filtered, smoothed, and normalized EMG data. The dataset of mean EMG amplitudes for each bench press set was assessed for normality by using a Shapiro Test on the residuals of the most-fitted ANOVA tests $(p<0.01)$, and by using quantile-quantile (Q-Q) plots. Based on these tests, it was unclear whether the mean EMG amplitude for each set was normally distributed. Thus, permutation tests were used to resample the mean EMG data, and permutation tests of symmetry and pairwise permutation tests were applied to comparisons for which the original test reported a significant difference. $P$-values for the pairwise permutation tests were adjusted using the Benjamini-Hochberg procedure to control for the false discovery rate due to multiple comparisons. ${ }^{14}$ In addition, bootstrap estimates using 1000 samples were performed to generate estimators and bias-corrected confidence intervals for both the general sample and for various comparisons. 


\section{Results}

Mean and median serratus anterior EMG amplitudes for each group and set are listed in Table 2. The order of sets was varied between the four groups; set numbers in Table 2 correspond to those in Table 1.

Table 2 Serratus anterior surface EMG amplitudes

\begin{tabular}{cccccccccc}
\hline & \multicolumn{4}{c}{ Set $^{*}$} & \multicolumn{2}{c}{ Set 2 } & \multicolumn{2}{c}{ Set 3 } & \multicolumn{2}{c}{ Set 4 } \\
Group & Side & Mean & Median & Mean & Median & Mean & Median & Mean & Median \\
\hline \multirow{2}{*}{1} & L & 41.7 & 39.7 & 44.9 & 42.2 & 55.6 & 55.5 & 66.9 & 63.0 \\
& R & 31.3 & 29.6 & 32.8 & 31.2 & 40.7 & 39.9 & 44.2 & 41.7 \\
& Avg & 36.5 & 34.6 & 38.8 & 36.7 & 48.2 & 47.7 & 55.6 & 52.4 \\
2 & L & 29.1 & 26.7 & 42.8 & 30.2 & 42.9 & 42.3 & 43.8 & 42.4 \\
& R & 36.1 & 31.2 & 29.0 & 27.3 & 41.3 & 39.1 & 43.8 & 41.7 \\
& Avg & 32.6 & 28.9 & 35.9 & 28.7 & 42.1 & 40.7 & 43.8 & 42.0 \\
3 & L & 36.9 & 34.2 & 34.6 & 32.4 & 43.9 & 41.1 & 55.2 & 51.5 \\
& R & 33.7 & 31.6 & 26.2 & 25.0 & 32.6 & 31.7 & 41.3 & 38.3 \\
& Avg & 35.3 & 32.9 & 30.4 & 28.7 & 38.2 & 36.4 & 48.3 & 44.9 \\
4 & L & 40.7 & 38.5 & 31.7 & 29.8 & 53.3 & 52.6 & 46.1 & 46.0 \\
& R & 28.2 & 26.3 & 23.5 & 22.1 & 38.4 & 38.5 & 34.7 & 33.0 \\
& Avg & 34.5 & 32.4 & 27.6 & 26.0 & 45.0 & 45.5 & 40.4 & 39.5 \\
\multicolumn{2}{|c}{ Overall Avg } & 34.7 & 32.2 & 33.2 & 30.0 & 43.6 & 42.6 & 47.0 & 44.7 \\
\hline
\end{tabular}

*Set numbers correspond to Table 1.

For all subjects, sets 1 and 2 used the lighter weight ( $43 \mathrm{~kg}$ ) and sets 3 and 4 used the heavier weight ( $61 \mathrm{~kg}$ ); as expected, higher EMG values were observed at the heavier weight (mean EMG amplitude, 47.340 versus 35.533; $p$ <.001). Mean EMG amplitudes for light and heavy barbell and dumbbell bench presses are shown in Table 3. Dumbbell presses showed greater mean EMG amplitude than barbell presses at both weights (total weight-normalized mean EMG amplitude: 44.742 versus 38.179, $p=.005$; mean EMG amplitude at $43 \mathrm{~kg}: 39.449$ versus 31.617, $p=.044$; mean EMG amplitude at $61 \mathrm{~kg}: 49.938$ versus $44.742, p=.025$ ).

Table 3 Mean EMG amplitude by bench press type and weight

\begin{tabular}{lc}
\hline Bench press type/weight & Mean \\
\hline Light barbell & 31.6 \\
Light dumbbell & 39.4 \\
Heavy barbell & 44.7 \\
Heavy dumbbell & 49.9 \\
Light $^{\mathrm{a}}$ & 35.5 \\
Heavy $^{\mathrm{a}}$ & 47.3 \\
Barbell $^{\mathrm{b}}$ & 38.2 \\
Dumbbell $^{\mathrm{b}}$ & 44.7 \\
\hline
\end{tabular}

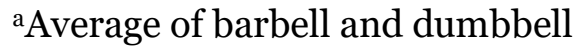

bAverage of light and heavy 
Since it was unclear whether the mean EMG amplitude distributions for our pooled samples were normally distributed, bootstrap estimates and confidence intervals were calculated to provide a more accurate estimate of the means of these values (Table 4), and a permutation test of symmetry was used to assess differences in mean EMG values between bench press variations and weights (Table 5).

Table 4 Bootstrapped mean EMG amplitudes and 95\% confidence intervals (CI)

\begin{tabular}{lcc}
\hline Bench press type/weight & Bootstrapped mean & $95 \% \mathrm{CI}$ \\
\hline Light barbell & 31.6 & $(27.3,37.0)$ \\
Light dumbbell & 39.5 & $(33.4,50.7)$ \\
Heavy barbell & 44.9 & $(38.6,51.9)$ \\
Heavy dumbbell & 50.1 & $(42.3,62.0)$ \\
Light $^{\mathrm{a}}$ & 35.5 & $(31.8,41.3)$ \\
Heavy $^{\mathrm{a}}$ & 47.2 & $(42.7,55.4)$ \\
Barbell $^{\mathrm{b}}$ & 38.3 & $(34.3,43.0)$ \\
Dumbbell $^{\mathrm{b}}$ & 44.7 & $(39.3,52.0)$ \\
\hline
\end{tabular}

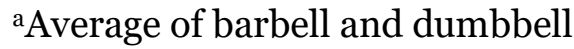

${ }^{b}$ Average of light and heavy

Table 5 Permutation test of symmetry

\begin{tabular}{cclc}
\hline & $\begin{array}{c}\text { Mean } \\
\text { EMG }\end{array}$ & \\
\# of factors & amplitude & Factor & $P$-value \\
\hline 2 & All & Exercise & 0.005 \\
2 & All & Weight & $<.001$ \\
4 & All & Exercise \& weight & $<.001$
\end{tabular}

Significant differences in mean EMG amplitude were observed between the following pairs: light barbell and light dumbbell, heavy barbell and heavy dumbbell, light and heavy (averages of barbell and dumbbell), and barbell and dumbbell (averages of light and heavy). Pairwise permutation tests were used to compare the mean EMG values; Table 6 shows the output of the test statistic corresponding to differences in mean EMG value and corresponding $p$-values. Table 7 shows bootstrapped estimates of differences between means, with biascorrected $95 \%$ confidence intervals.

Table 6 Pairwise permutation tests: Test statistic ( $P$-value)

\begin{tabular}{llll}
\hline & Heavy barbell & Light barbell & Heavy dumbbell \\
\hline Light barbell & $5.28(<.001)$ & & \\
Heavy dumbbell & $-2.09(.044)$ & $-4.48(<.001)$ & \\
Light dumbbell & $1.80(.072)$ & $-2.40(.025)$ & $2.57(.020)$
\end{tabular}


Table 7 Bootstrapped estimates of differences between mean EMG values (bias-corrected 95\% confidence intervals)

\begin{tabular}{llll} 
& Heavy barbell & Light barbell & Heavy dumbbell \\
\hline Light barbell & $0.877(0.505,1.17)$ & & \\
Heavy dumbbell & $\begin{array}{l}-0.138(-0.428, \\
0.143)\end{array}$ & $\begin{array}{l}-0.633(-0.806,- \\
0.310)\end{array}$ & \\
& & $-0.255(-0.479,0.073)$ & $0.414(-0.124,0.794)$
\end{tabular}

\section{Discussion}

In this study we examined whether barbell and dumbbell bench press elicit different degrees of serratus anterior muscle activation. Dumbbell bench press sets elicited significantly greater serratus anterior activity than barbell press sets at both weights tested (43 and $61 \mathrm{~kg}$ ). Because of the importance of the serratus anterior to scapula stability and mobility, these results suggest that dumbbell press may be preferable to barbell press for upper-body strength training by overhead throwing athletes.

One limitation of this study is the large amount of noise that was associated with the EMG signals. ${ }^{15}$ To minimize the effect of noise on data analysis, each subject followed the same rest period between sets, and was provided the same instructions regarding technique, including minimizing movement or muscle recruitment unrelated to the exercise. Following data collection, filtering, smoothing, normalizing, and amplitude extraction methods were used to separate signal from noise. ${ }^{16}$

The results indicate that the more unstable of the two bench press exercises-dumbbell press-resulted in greater serratus anterior muscle activation. These results are consistent with previous studies that used surface EMG analysis to measure serratus anterior activity during bench press. Caravan et al. found that the ratio of serratus anterior to upper trapezius activity was greater during push-ups than during the more stable barbell bench press. ${ }^{6}$ Similarly, Tucci et al. found that serratus anterior activity was greater during wall press than during barbell press. ${ }^{17}$ Other upper-body muscles also show greater activity during less stable bench press exercises based on surface EMG analysis. For example, the triceps, upper trapezius, and gluteus maximus exhibited greater activity during suspended push-ups than during more stable standard push-ups. ${ }^{18}$

Interestingly, pre-activation of the serratus anterior and other muscles (pectoralis major, deltoid anterior, and triceps brachii) by bench press can cause greater muscle activation during subsequent sets. ${ }^{20}$ Baker et al. found that power output in explosive bench press throws was greater when preceded by a heavy bench press set. ${ }^{19}$ To avoid a systemic muscle pre-activation effect, we varied the order of the four sets, with the two two lights sets followed by the two heavy sets.

Future studies of serratus anterior activation during bench press should investigate the effect of muscle pre-activation, using a greater range of weights, and should control for differences in trunk inclination and hand spacing. Barnett et al. measured the activity of five shoulder muscles using surface EMG at four trunk inclinations and two hand spacings, and showed that both of these variables had a significant effect on muscle activity. ${ }^{21}$ Another important variable is whether muscle-specific instructions on technique are provided to subjects before exercises. Daniels et al. showed that triceps brachii activity varied significantly depending on whether instructions were provided. ${ }^{22}$ Subjects also use different movement patterns during 
bench press exercises, and these differences are likely to cause differences in muscle activation. ${ }^{23}$ Instructions that ensure more uniform movement patterns are desired.

\section{Practical Applications}

Overhead throwing athletes like baseball players are subject to high forces and torques during their sports-specific performance. General rehabilitation and injury-preventive exercises are highly sought after to reduce the impact of performance on arm and general physical health. The statistically robust finding that the dumbbell bench press variation elicits more serratus anterior muscle activation is of great value to anyone interested in designing exercise routines or programming for overhead athletes.

\section{Conclusion}

Dumbbell bench press elicited greater serratus anterior muscle activation than barbell bench press at both weights tested. These results provide insight for overhead throwing athletes into the selection of bench press exercise for serratus anterior strengthening and for general arm health.

\section{Acknowledgments}

The authors thank Michael O'Connell, Anthony Brady, and Joseph Marsh for editing and reviewing this paper.

\section{References}

1. Cools AM, Witvrouw EE, Declercq GA, Vanderstraeten GG, Cambier DC. Evaluation of isokinetic force production and associated muscle activity in the scapular rotators during a protraction-retraction movement in overhead athletes with impingement symptoms. Br J Sports Med. 2004;38(1):64-68.

2. Kibler WB. The role of the scapula in athletic shoulder function. Am J Sports Med. 1998;26(2):325-337.

3. Mottram SL. Dynamic stability of the scapula. Man Ther. 1997;2(3):123-131.

4. Schick EE, Coburn JW, Brown LE, Judelson DA, Khamoui AV, Tran TT, Uribe BP. A comparison of muscle activation between a Smith machine and free weight bench press. $J$ Strength Cond Res. 2010;24(3):779-784.

5. Decker MJ, Hintermeister RA, Faber KJ, Hawkins RJ. Serratus anterior muscle activity during selected rehabilitation exercises. Am J Sports Med. 1999;27(6):784-791.

6. Martins J, Tucci HT, Andrade R, Araújo RC, Bevilaqua-Grossi D, Oliveira AS. Electromyographic amplitude ratio of serratus anterior and upper trapezius muscles during modified push-ups and bench press exercises: $J$ Strength Cond Res. 2008;22(2):477-484.

7. Caravan A, Scheffey JO, Briend SJ, Boddy KJ. Surface electromyographic analysis of differential effects in kettlebell carries for the serratus anterior muscles. PeerJ. 2018;6:e5044.

8. Ekstrom RA, Soderberg GL, Donatelli RA. Normalization procedures using maximum voluntary isometric contractions for the serratus anterior and trapezius muscles during surface EMG analysis. J Electromyogr Kinesiol. 2005;15(4):418-428. 
9. De Luca G. Fundamental concepts in EMG signal acquisition. Delsys Inc; 2003.

10. De Luca CJ, Donald Gilmore L, Kuznetsov M, Roy SH. Filtering the surface EMG signal: Movement artifact and baseline noise contamination. $J$ Biomech. 2010;43(8):1573-1579.

11. Dieterich AV, Botter A, Vieira TM, Peolsson A, Petzke F, Davey P, Falla D. Spatial variation and inconsistency between estimates of onset of muscle activation from EMG and ultrasound. Sci Rep. 2017;7:42011.

12. Boettcher CE, Ginn KA, Cathers I. Standard maximum isometric voluntary contraction tests for normalizing shoulder muscle EMG. J Orthop Res. 2008;26(12):1591-1597.

13. Halaki M, Ginn K. Normalization of EMG signals: To normalize or not to normalize and what to normalize to? In: Naik GR, ed. Computational Intelligence in Electromyography Analysis - A Perspective on Current Applications and Future Challenges. London: InTech Open Access Publisher; 2012:175-194.

14. Benjamini $Y$, Hochberg Y. Controlling the false discovery rate: a practical and powerful approach to multiple testing. $J$ R Stat Soc Series B 1995;57(1):289-300.

15. Vigotsky AD, Beardsley C, Contreras B, Steele J, Ogborn D, Phillips SM. Greater electromyographic responses do not imply greater motor unit recruitment and 'hypertrophic potential' cannot be inferred. $J$ Strength Cond Res. 2015; 31(1):e1-e4.

16. Ozkaya U, Coskun O, Comlekci S. 2010. Frequency analysis of EMG signals with Matlab sptool. In: Proceedings of the 9th WSEAS International Conference on Signal Processing, Robotics, and Automation. Stevens Point, WI: World Scientific and Engineering Academy and Society; 2010:83-88.

17. Tucci HT, Ciol MA, De Araújo RC, De Andrade R, Martins J, McQuade KJ, Oliveira AS. Muscle activation of selected shoulder muscles during unilateral wall and bench press tasks under submaximal isometric effort. J Orthop Sports Phys Ther. 2011;41(7):520525.

18. Calatayud J, Borreani S, Colado JC, Martin F, Batalha N, Silva A. Muscle activation differences between stable push-ups and push-ups with a unilateral v-shaped suspension system at different heights. Motricidade 2014;10(4):84-93.

19. Baker D. Acute effect of alternating heavy and light resistances on power output during upper-body complex power training. $J$ Strength Cond Res. 2003;17(3):493-497.

20. Pirauá A, Barros Beltrão N, Ximenes Santos C, Pitangui A, Araújo R. Analysis of muscle activity during the bench press exercise performed with the pre-activation method on stable and unstable surfaces. Kinesiology 2017;49(2). DOI: 10.26582/k.49.2.11.

21. Barnett C, Kippers V, Turner P. Effects of variations of the bench press exercise on the EMG activity of five shoulder muscles. The Journal of Strength \& Conditioning Research 1995; $9(4): 222-227$.

22. Daniels RJ, Cook SB. Effect of instructions on EMG during the bench press in trained and untrained males. Hum Mov Sci. 2017;55:182-188.

23. Vieira TM, Bisi MC, Stagni R, Botter A. Changes in tibialis anterior architecture affect the amplitude of surface electromyograms. J Neuroeng Rehabil. 2017;14(1):81. 